5. Über gehaltene Referate zu unserem Arbeitsgebiet, wichtige Diskussionsvoten und vor allem konkrete Schlußresultate werden die Vereinsmitglieder durch die «Geographica Helvetica » orientiert. Zuerst wird das Thema «Die geographischen Namen im Unterricht » behandelt. Die da und dort geübte Art des Umgangs mit diesen Namen war es in erster Linie, welche die Geographie immer wieder in Mißkredit brachte, und wenn es möglich wird, zu einer allgemein anerkannten Lösung des Namen-Problems zu gelangen, dann scheint uns schon viel gewonnen zu sein. Die beiden Lehrer für Geographie an der Kant. Handelsschule Zürich, Dr. R. Merian und Dr. W. NigG, legen einen Lösungsversuch vor, den sie gemeinsam erarbeiteten und über den Dr. Merian in Olten berichtet hat. Die Mitglieder sind gebeten, sich möglichst bald dazu zu äußern.

6. Die vorgeschlagenen Programmpunkte ersetzen die bisherige Tätigkeit des Vereins nicht, sondern sind zusätzlich zu dieser gedacht. Auch das Exkursionsprogramm ist mit Absicht umfangreich gestaltet worden.

Für den Vorstand: O. WERNLI

\title{
DIE GEOGRAPHISCHEN NAMEN IM UNTERRICHT
}

\section{Rudolf Merian und Werner Nigg}

Wie oft stellt man als Lehrer fest, daß es immer wieder Schüler gibt, die beim Repetieren versagen, weil sie keine richtige Vorstellung des zu besprechenden Landes besitzen und viele geographische Namen vergessen haben. Dieses Versagen der Schüler ist manchmal umso unverständlicher, als ja diese Namen im Unterricht sicher einmal erwähnt wurden und Atlas wie Schulwandkarte meistens vor den Augen der Schüler sind.

$\mathrm{Da}$ leider viele, z. T. einflußreiche Persönlichkeiten zu Unrecht auch heute noch Geographie mit Namenlernen gleichstellen, und dies unserem Fache schadet, wurde wohl allgemein einer systematischen Behandlung der geographischen Namen im Unterricht in der letzten Zeit zu wenig Beachtung geschenkt. Und doch ist es gerade heute wichtig, daß jeder Mittelschüler auch über ein bestimmtes Namenwissen verfügt. Erstes Erfordernis ist allerdings, daß man sich immer bewußt bleibt, daß das Erlernen geographischer Namen nicht Selbstzweck sein darf. Es muß Mittel zum Zwecke bleiben, wie das Lernen der Wörter bei Fremdsprachen.

Versuche einer systematischen Behandlung der geographischen Namen sind nicht neu. Schon unser Vorgänger, Prof. Dr. E. Wettrer, wie auch Prof. Dr. H. Gutersohn, W. Angst u. a. haben in dieser Richtung wertvolle Arbeit geleistet.

Wir haben nun an der Kant. Handelsschule Zürich seit 4 Jahren eine Methode ausprobiert und immer wieder verbessert, die wir hier unseren Kollegen als Diskussionsgrundlage unterbreiten. Dabei sei ausdrücklich festgehalten, daß sie keineswegs als endgültig erprobt betrachtet wird, und $d a ß$ die ausgewählten Namen für eine Handelsschule mit 3 Jahren Geographieunterricht gedacht sind. Wir wären allen Kollegen dankbar, wenn sie an dieser Methode - vor allem an der Auswahl der Namen - sachliche Kritik übten, und wenn sie uns Verbesserungs- und Änderungsvorschläge mitteilen würden.

Es wurde wie folgt vorgegangen:

1. Wir teilen den Schülern Namenblätter mit einigen hundert geographischen Namen aus, geordnet nach den Ausschnitten der verschiedenen Westermannstempel. Die Namen sind nach der Bedeutung der durch sie bezeichneten geographischen Objekte ausgewählt. Die Schreibweise ist vorwiegend die der deutschsprachigen Ausgabe des Schweiz. Mittelschulatlasses. 
2. Jeder einzelne dieser Namen wird mit den Schülern im Verlaufe des ersten Schuljahres (15-16 jährige Schüler) an der Wandkarte und an projizierten Stempeln besprochen, so daß dem Schüler zugleich mit dem Namen Lage und Bedeutung des benannten Objekts klar wird. Als Hausaufgabe tragen die Schüler die Namen in den Stempel eines besonderen Namenheftes ein, zeichnen eine einfache Karte, und lernen so zugleich Namen, Lage und Bedeutung der geographischen Objekte.

3. Laufend werden während der ersten Klasse diese Namen mündlich oder schriftlich repetiert. In allen folgenden Klassen finden immer wieder angemeldete oder überraschende Repetitionen des gesamten Namenstoffes statt. Es ist jedem einzelnen Lehrer zu überlassen, diese Repetitionen so abwechslungsreich wie möglich zu gestalten. So läßt man z. B. das eine Mal die Namen auswendig in einen Stempel zeichnen, ein anderes Mal verlangt man die Beschreibung von Lage und Bedeutung, oder stellt die Namen zu Begriffspaaren zusammen usw.

Dieses Vorgehen hat nach unserer Meinung namentlich folgende Vorteile:

1. Die geographischen Namen werden systematisch erlernt, der Schüler weiß, daß er diesen Grundstock von Namen kennen muß.

2. Der Schüler erhält eine geographische Übersicht über die ganze Erde, auch von solchen Ländern, die man im Unterricht nicht besprechen kann.

3. Das Lernen ist keine rein mechanische Sache, denn der Schüler wird dazu angehalten mit dem Namen der Objekte zugleich deren Bedeutung und Lagebeziehungen zu erfassen und auch selbst möglichst klar zu umschreiben.

\section{VERZEICHNIS DER NAMEN}

Die Gruppierung und Anordnung konnte nicht streng systematisch erfolgen; sie richten sich nach den praktischen Erfahrungen mit den Westermannstempeln. So ist z. B. die Begrenzung durch den Stempel gegeben; ferner wurden oft zusammengehörige Namen auf verschiedene Stempel verteilt, um diese nicht zu "überladen“.

Stempel Erde. Europa, Asien, Afrika, Nordamerika, Südamerika, Australien, Arktis, Antarktis, Atlantischer Ozean, Pazifik oder Großer- oder Stiller Ozean, Indischer Ozean, Nördliches und Südliches Eismeer.

Stempel Europa. Alpen, Jura, Apennin, Dinarische Alpen, Karst, Pindus, Karpaten, Transsilvanische Alpen, Balkan, Rhodope Gebirge, Pyrenäen, Kastilisches Scheidegebirge, Sierra Morena, Sierra Nevada, Zentralplateau, Mitteldeutsches Bergland, Skandinavisches Gebirge, Ural, Island, Reykjavik, Spitzbergen.

Norddeutsches Tiefland, Poebene, Ober- und Niederungarische Tiefebene, Walachei, Schwäbischbayrische Hochebene.

Nordsee, Doggerbank, Ostsee, Bottnischer Meerbusen, Finnischer Meerbusen, Kanal, Golf von Vizcaya, Mittelländisches Meer, Tyrrhenisches Meer, Ligurisches Meer, Adriatisches Meer, Ionisches Meer, Aegäisches Meer, Schwarzes Meer, Asowsches Meer.

Rhein, Mosel, Main, Maas, Weser, Elbe, Oder, Weichsel, Seine, Loire, Garonne, Rhone, Saône, Po, Donau, Inn, Theiß, Save, Ebro, Guadalquivir, Tajo, Dueró, Wolga, Don, Donez, Dnjepr.

Stempel Frankreich. Paris, Ile de France, Champagne, Reims, Lothringen, Nancy, Elsaß, Straßburg, Mühlhausen, Vogesen, Burgund, Plateau de Langres, Dijon, Lyon, Savoyen, Mt. Blanc, Mt. Cenis, Languedoc, Marseille, Sète, Provence, Riviera, Nizza, Monaco, Auvergne, Clermont Ferrand, St-Etienne, Cevennen, Mt. Dore, Bordeaux, Toulouse, Landes, Biarritz, Andorra, Orléans, Nantes, St-Nazaire, Bretagne, Brest, Nordmandie, Le Havre, Rouen, Artois, Picardie, Lille, Calais, Dunkerque.

Belgien, Bruxelles, Flandern, Antwerpen, Liège, Ardennen, Luxemburg, Saargebiet, Saarbrücken.

Stempel Großbritannien und Irland. England, London, Schottland, Wales, Irland, Nordirland, Irischer Freistaat, Dublin, Themse, Strasse von Dover, Southampton, Cornwall, Bristol, Birmingham, Manchester, Liverpool, Leeds, Sheffield, Newcastle, Edinburgh, Glasgow, Belfast.

Stempel Niederlande. Amsterdarn, Holland, Zeeland, Friesland, Limburg, Rotterdam, Den Haag, Utrecht, Zuidersee.

Stempel Deutschland. Bundesrepublik Deutschland, Bonn, München, Nürnberg, Stuttgart, Neckar, Schwarzwald, Oberrheinische Tiefebene, Frankfurt, Mannheim, Karlsruhe, Rheinland, Köln, Rheinisches Schiefergebirge, Ruhrgebiet, Düsseldorf, Essen, Duisburg, Dortmund, Hannover, Braunschweig, Harz, Bremen, Hamburg. Friesische Inseln, Kiel. Deutsche Demokratische Republik, Berlin, Sachsen, Leipzig, Dresden, Thüringen, Weimar.

Polen. Warschau, Krakau, Schlesien, Breslau, Danzig.

Tschechoslovakei. Prag, Böhmen, Pilsen, Erzgebirge, Sudeten, Mähren, Brünn, Slovakei, Bratislava.

Oesterreich. Wien, Salzburg, Linz, Tirol, Innsbruck, Arlberg, Vorarlberg. 
Stempel Skandinavien. Dänemark. Kopenhagen, Seeland, Fünen, Jütland, Sund, Großer und Kleiner Belt.

Schrweden. Stockholm, Uppsala, Göteborg, Malmö, Lappland, Kiruna, Lulea.

Norzwegen. Oslo, Bergen, Sognefjord, Hardangerfjord, Trondheim, Lofoten, Westfjord, Narvik, Hammerfest.

Finnland. Helsinki, Turku, Finnische Seenplatte.

Baltische Staaten. Estland, Lettland, Riga, Litauen.

Stempel Spanien. Madrid, Altkastilien, Neukastilien, Galicien, Baskische Provinzen, Bilbao, San Sebastian, Aragonien, Katalonien, Barcelona, Valencia, Murcia, Malaga, Granada, Andalusien, Sevilla, Córdoba, Cádiz, Gibraltar, Balearen, Tanger, Span. Marokko.

Portugal. Lissabon, Porto.

Stempel Italien. Rom, Piemont, Turin, Lombardei, Mailand, Emilia, Bologna, Venetien, Venedig, Triest, Verona, Gardasee, Südtirol, Etsch, Brenner, Ligurien, Genua, Toscana, Florenz, Arno, Maremmen, Abruzzen, Umbrien, Tiber, Neapel, Vesuv, Apulien, Bari, Calabrien, Sizilien, Palermo, Catania, Messina, Aetna, Stromboli, Sardinien, Korsika.

Stempel Balkanhalbinsel. Ungarn. Budapest, Pussta.

Rumänien. Bukarest.

Bulgarien. Sofia.

Albanien. Tirana.

Jugoslarvien. Belgrad, Zagreb, Kroatien, Dalmatien, Dubrovnik, Montenegro.

Griechenland. Athen, Piräus, Makedonien, Saloniki, Peloponnes, Ionische Inseln, Kykladen, Sporaden, Santorin, Rodos, Kreta.

Türkei. Istanbul, Bosporus, Marmarameer, Dardanellen, Smyrna, Mäander.

Stempel Rußland. Moskau, Leningrad, Murmansk, Archangelsk, Weißrußland, Ukraine, Kiew, Charkow, Rostow, Odessa, Krim, Stalingrad, Astrachan, Kaukasus, Elbrus, Baku, Tiflis, Batum, Kaspisches Meer, Magnitogorsk.

Stempel Vorderasien. Tiirkei. Ankara, Kleinasien, Anatolien, Cypern, Ararat.

Iran. Teheran, Isfahan, Täbris, Abadan.

Libanon. Beirut.

Syrien. Damaskus, Aleppo.

Israel. Palästina. Jerusalem, Tel Awiw, Haifa, Totes Meer.

Jordanien. Ammán, Jordan.

Irak. Bagdad, Mesopotamien, Euphrat, Tigris, Schatt el Arab, Mosul, Kerkuk, Basra.

Arabien. Saud Arabien, Er Riad, Mekka, Medina, Bahrein Koweit.

Aegypten. Kairo, Alexandria, Port Said, Sues, Sueskanal, Assuan, Siwah.

Stempel Vorder- und Hinterindien. Indus, Ganges, Bramaputra, Satletsch, West- und Ostghats, Dekan, Thar.

Indien. (Indische Union) Delhi, Benares, Calcutta, Bengalen, Assam, Tscherrapunchi, Malabar-

küste, Bombay, Goa, Koromandelküste, Madras, Hyderabad.

Pakistan. (West- und Ostpakistan) Karachi, Lahore, Punjab, Ostbengalen.

Himalaya. Mt. Everest, Darjeeling, Nepal, Kathmandu, Kaschmir.

Afghanistan. Kabul, Khaibarpaß.

Burma. Rangoon, Irawadi, Salween.

Thailand. (Siam) Bangkok, Menam.

Brit. Malaya, Singapore.

Indochina. Mekong, Viet Nam, Saigon, Viet Minh, Tonking, Hanoi, Cambodja, Laos.

Stempel Asien. Pontinisches Gebirge, Taurus, Armenien, Hochland von Iran, Elburs, Hindu

kusch, Pamir, Himalaya, Transhimalaya, Kwenlun, Tienschan, Altai, Sajanisches Gebirge, Chingan, Südchinesisches Bergland, Malayscher Archipel; Rotes Meer, Persischer Golf, Arabisches Meer, Bengalisches Meer, Südchinesisches Meer, Ostchinesisches Meer, Gelbes Meer, Japanisches Meer, Ochotskisches Meer, Beringmeer; Aden, Jemen, Hadramut, Oman.

Indonesien. Grosse Sundainseln, Java, Batavia, (Djakarta) Krakatau, Sumatra, Palembang, Borneo,

Celebes, Kleine Sundainseln, Bali, Molukken, Neu Guinea.

Philippinen. Luzon, Manila, Mindanao, Philippinengraben.

China. (Eigentliches China) Hwang-ho, Jang-tse-kiang, Hsi-kang, Peking, Tientsin, Schanghai, Nanking, Hankau, Tschungking, Kanton, Hong-kong, Formosa.

Mandschurei. Mukden, Dairen.

Korea. Südkorea, Söul, Nordkorea.

Japan. Tokio, Hondo, Schikoku, Kiuschiu, Hokkaido, Riukiu, Yokohama, Osaka, Kioto, Kobe, Nagasaki.

Innerasien. Tibet, Lhasa, Ostturkestan, Tarim, Kaschgar, Mongolei, Gobi, Aeussere Mongolei, Dsungarei.

Sibirien. Ob, Irtysch, Nowo Sibirsk, Kusnetzk, Jenissei, Irkutsk, Baikalsee, Lena, Taimyrhalbinsel, Werchojansk, Tschuktschen Halbinsel, Kamtschatka, Ferner Osten, Amur, Wladiwostok, Sachalin, Kurilen. 
West-Turkestan. Kaspisches Meer, Aralsee, Amu Darja, Syr Darja, Taschkent, Samarkand, Kirgisensteppe.

Stempel Afrika. Nord-Afrika, Atlasländer, Atlas, Marokko, Rabat, Marrakesch, Casablanca, Fez, Algerien, Algier, Oran, Constantine, Tunesien, Tunis, Malta.

Libyen. Tripolis; Kanarische Inseln, Madeira.

Sahara. Hoggar, Tibesti, In Salah, Biskra, Kufra.

Sudan. Französisch Westafrika, Dakar, Senegal, Tombouctou, Tschadsee, Engl. Aegyptischer Sudan, Khartum, Nil (Weißer und Blauer Nil), Atbara, Sobat, Nubien.

Ober Guinea. Sierra Leone, Liberia, Goldküste, Accra, Nigerien, Lagos, Kano, Fernando Poo, Sao Thomé, Kamerun, Kamerunberg.

Zentralafrika. Belg. Kongo, Léopoldville, Elisabethville, Katanga, Kongo, Kassai, Ubangi, Französisch Aequatorialafrika, Brazzaville, Lambaréné.

Ostafrika. Abessinien, Addis Abeba, Harar, Erythräa, Französisch Somaliland, Djibouti, Srasse von Bab el Mandeb, Britisch Somaliland, Italienisch Somaliland; Kenia, Nairobi, Uganda, Viktoriasee, Kilimandscharo, Tanganjika Terr., Dar-es-Salaam, Zanzibar, Tanganjikasee, Kiwusee, Eduardsee, Albertsee, Ruwenzori; Madagaskar.

Stempel Südafrika. Angola, Moçambique, Lourenço Marques, Nordrhodesia, Südrhodesia, Viktoriafälle, Kalahari.

Siidafrikanische Union. Pretoria, Kapland, Kapstadt, Natal, Durban, Oranje Freistaat, Oranje, Transvaal, Johannesburg, Kimberley, SW-Afrika, Drakensberge.

Stempel Nordamerika. Rocky Mountains, Kaskadengebirge, Sierra Nevada, Columbiaplateau, Großes Becken, Coloradoplateau, Wahsatchgebirge; Mittelwesten, Prärien, Appalachen, Hudsonbay, Golf von Mexico, Colorado, Columbia, Mississippi, Missouri, Ohio, Rio Grande del Norte, Hudson, Mackenzie, Nelson.

Alaska. Mt. McKinley, Aleuten, Yukon.

Kanada. Ottawa, Neufundland, St. Johns; Prinz Eduard Insel; Neu Schottland, Halifax; Neu-Braunschweig; Quebec, Montreal, St. Lorenz-Strom; Ontario, Toronto; Manitoba, Winnipeg; Saskatchewan, Regina; Alberta, Edmonton, Calgary; Britisch Columbia, Victoria, Vancouver; Nordwestterritorien; Großer Bärensee, Großer Sklavensee. Grönland.

Mexiko. Mexiko City, Tampico, Sierra Madre Oriental, Sierra Madre Occidental; Popocatepetl.

Stempel USA. Washington; Neuenglandstaaten, Boston; Mittelatlantische und Südatlantische Staaten, New York, New Yersey, Pennsylvanien, Philadelphia, Pittsburgh, Maryland, Baltimore, District of Columbia, Virginia, Carolina, Georgia, Atlanta, Florida, Miami; Zentralstaaten, Oberer See, Michigan-See, Huron-See, Erie-See, Ontario-See, Niagara Falls, Sault Ste. Marie, Duluth, Illinois, Chicago, Detroit, Cleveland, Buffalo, St. Paul, Minneapolis, Kansas City, Louisiana, New Orleans, Alabama, Birmingham, Texas, Houston; Gebirgs- und Pazifik-Staaten, California, Los Angeles, San Francisco, Seattle; Großer Salzsee, Salt Lake City, Denver.

Stempel Mittelamerika. Große Antillen, Cuba, Habana, Haïti, San Domingo, Puerto Rico, Jamaica, Bahamainseln; Kleine Antillen, Martinique, Trinidad; Yukatan, Guatemala, El Salvador, Brit. Honduras, Honduras, Nicaragua, Costa Rica, Panama, Panamakanal, Kanlazone.

Stempel Südamerika. Kordilleren oder Anden, Aconcagua, Brasilianisches Bergland, Hochland von Guayana, Llanos, Selvas, Gran Chaco, Pampas, Patagonien, Feuerland, Falklandinseln, Amazonas, Kio Negro, Rio Madeira, Orinoco, Rio Magdalena, Rio Cauca, Paranà, Paraguay, Rio de la Plata, Titicacasee.

Brasilien. Rio de Janairo, Sao Paolo, Santos, Rio Grande do Sul, Minas Geraes, Bello Horizonte, Bahia, Pernambuco, Belem, Manaos.

La Plata Länder. Argentinien, Buenos Aires, Rosario, Bahia Blanca, Mendoza.

Uruguay. Montevideo.

Paraguay. Asunciòn.

Andenländer. Chile. Santiago de Chile, Valparaiso, Atàcamawüste.

Bolivien. La Paz, Potosi.

Peru. Lima, Cuzco, Cerro del Pasco.

Ecuador. Quito.

Kolumbien. Bogotà, Buenaventura.

Venezuela. Caràcas, Maracaibo, Britisch-, Niederländisch-, Französisch-Guayana.

Stempel Australien. Canberra, Murray, Darling, Neu Südwales, Sydney, Newcastle, Victoria, Melbourne, Tasmanien, Südaustralien, Adelaide, Westaustralien, Perth, Nordaustralien, Queensland, Brisbane.

Stempel Ozeanien. Neuseeland, Nordinsel, Wellington, Auckland, Südinsel, Mt. Cook.

Melanesien, Mikronesien, Polynesien, Samoa, Fidschi-Inseln, Tahiti, Sandwich Inseln, Hawaii, Honolulu, Mauna Loa. 